Vol 12, Issue 9, 2019

\title{
THE ROLE OF ASTAXANTHIN COMPARED WITH METFORMIN IN PREVENTING GLYCATED HUMAN SERUM ALBUMIN FROM POSSIBLE UNFOLDING: A MOLECULAR DYNAMIC STUDY
}

\author{
SYAHPUTRA WIBOWO ${ }^{1}$, SRI WIDYARTI ${ }^{1}$, AKHMAD SABARUDIN ${ }^{2}$, DJOKO WAHONO SOEATMADJI ${ }^{3}$ \\ SUTIMAN BAMBANG SUMITRO ${ }^{1 *}$
}

\begin{abstract}
${ }^{1}$ Department of Biology, Faculty of Mathematics and Natural Sciences, Brawijaya University Jl. Veteran, Malang 65145, East Java, Indonesia. ${ }^{2}$ Department of Chemistry, Faculty of Mathematics and Natural Sciences, Brawijaya University, Jl. Veteran, Malang 65145, East Java, Indonesia. ${ }^{3}$ Department of Internal, School of Medicine, Brawijaya University, Jl. Veteran, Malang 65145, East Java, Indonesia.
\end{abstract} Email: sutiman@ub.ac.id

Received: 08 July 2019, Revised and Accepted: 30 July 2019

ABSTRACT

Objectives: Albumin in diabetes mellitus undergoes conformational changes that affect the ability as an endogenous scavenger. Treatment with astaxanthin (ASX) expected to improve the function of albumin in case of diabetes mellitus. The objectives of this study are to compare the capability of ASX and metformin to prevent conformational changes on glycated albumin.

Methods: Data mining is performed to obtain human serum albumin (HSA) (4K2C), glucose (79025), ASX (5281224), and metformin (4091). Data preparation used PyRx and Discovery Studio 2016 Client. PyRx is utilized for docking and analysis of receptor-ligand interactions with LigPlus and Discovery Studio 2016 Client. YASARA is used for molecular dynamics simulations with a running time of $15.000 \mathrm{ps}$.

Results: A description of the glycated-HSA (gHSA) conformational changes that are bound to metformin has been successfully carried out. Changes that occur were unfolding and release of bonds in gHSA. Unfolding on gHSA includes the release of bonds between sites A and B. The root mean square deviation (RMSD) backbone value of metformin-gHSA shows a significant difference with gHSA at 8650 ps where gHSA showed $6.47 \mathrm{~nm}$ while the metformin-gHSA was $8.06 \mathrm{~nm}$ and continues to increase up to $15.72 \mathrm{~nm}$ at the end of the simulation. RMSD and root mean square fluctuation residues of gHSA which were interacted with ASX showed conditions close to normal HSA. In 11725 ps ASX-gHSA remained stable at $5.78 \mathrm{~nm}$, whereas gHSA increased to $8.13 \mathrm{~nm}$. gHSA at the end of the simulation showed a number of $9.052 \mathrm{~nm}$ while the normal HSA was $7.561 \mathrm{~nm}$.

Conclusion: This result indicated that ASX prevents gHSA from possible unfolding.

Keywords: Astaxanthin, Glycated human serum albumin, Metformin, Molecular Dynamic, Root mean square deviation, Root mean square fluctuation.

(c) 2019 The Authors. Published by Innovare Academic Sciences Pvt Ltd. This is an open access article under the CC BY license (http://creativecommons. org/licenses/by/4. 0/) DOI: http://dx.doi.org/10.22159/ajpcr.2019.v12i9.34617

\section{INTRODUCTION}

Diabetes mellitus is a degenerative disease that tends to experience a complication due to the change of physicochemical of blood [1]. Main characteristics of the changes in physicochemical of blood are a change in the rate of blood viscosity which is very related to the functional aspect of albumin as a transporter and scavenger. In diabetes mellitus case is likely to increase free radicals [2].

Free radicals are normal component in cellular metabolism, but if the amount of excess free radicals can cause cell damage that impacts oxidative as well as molecular and is a major factor causes the emergence of pathological conditions. This oxidative damage causes a conformational change of the protein that is often found in cases of diabetes and a variety of degenerative diseases. Albumin in type 2 diabetes mellitus (T2DM) undergoes conformational changes that affect the ability as an endogenous scavenger [3]. Human serum albumin (HSA) has the same properties as other biological macromolecules which have a dynamic nature and not rigid. This allows to study the function of HSA physiologically. Alzheimer's disease is an example of which is the implication of folding [4]. Albumin that is synthesized by hepatocytes of the liver as well as circulated continuously has a total of $60 \%-65 \%$ of the total plasma protein [5].

The presence of albumin in the blood of human beings is inseparable from its ability to bind to a wide variety of molecules such as fatty acids, metal ions or hormones, and drugs. There are two domains in the protein albumin, which has a high level of flexibility against the binding of the ligand, called as Sudlow's Sites I and II [6]. These domains related to the ability of albumin as a scavenger which free radical-trapping is run by a single cysteine residue (Cys34) where as many as $70-80 \%$ of the residues in adults contain sulfhydryl group [3].

Free radicals are formed in general that is the hydroxyl radical produced from copper or iron ions when reacted with oxygen. Final results from the presence of free radicals, i.e., the occurrence of oxidative stress on the body's proteins include albumin [3]. According to Otagiri and Chang [5], protein modification can occur through certain pathological circumstances. One of the causes of damage to protein albumin, i.e., if exposed to AGE (advanced glycation end-products).

The process of glycation can aggravate the condition of diabetes mellitus, where glycation agents such as galactose, ribose, and glyceraldehyde accelerate the glycation process and also have detergent effect on albumin which is unfolding and the opening of the hydrophobic surface, also amyloid formation on the structure of HSA [7]. Then, the modification site albumin that has occurred on glycated albumin including K199, K281, and N-terminus and the presence of Lys281, Arg428, Lys199, Arg98, Arg19, Arg521, Lys93, Lys276, Lys286, Lys414, Lys439, and Lys524 [8].

Treatment on the diabetes mellitus type 2 patients is generally given metformin [9]. Metformin can lower glucose levels in the blood. Metformin is the first line of treatment for sufferers of T2DM. This drug 
has the ability to be used in conjunction with other diabetes drugs due to efficacy and safety [10]. Metformin can decrease hepatic glucose in T2DM patient and improve peripheral glucose uptake [11]. Molecular mechanism of metformin related to activation of AMP-activated protein kinase and protein kinase A also can cause the inhibition from the mitochondrial respiratory chain (complex 1) and glycerophosphate dehydrogenase. This drug can increase metabolism such as insulin resistance and hyperglycemia with lead to reducing chronic inflammatory responses [12]

Metformin has side effects such as vomiting and nausea, even the most serious case is lactic acidosis state, especially in T2DM patients with kidney and liver dysfunction [13]. This research using a new perspective based on physicochemical properties of the glycated albumin. One of the therapeutic agents into a compelling study in this research is astaxanthin (ASX). ASX is a red carotenoid pigment, which does not have the activity of provitamin $\mathrm{A}$, and a biological antioxidant that occurs naturally in many types of plants, algae, and seafood. ASX has a range of pharmacological activity, including antioxidant activity, anti-inflammatory, immunomodulatory, anticancer, and antidiabetic. Inside the cell, ASX can effectively erodes and destroys the lipid peroxide radicals, thus, protecting the biological membrane fatty acids from oxidative damage [14]. Treatment with ASX expected to improve the function of albumin in case of diabetes mellitus. Molecular dynamics simulation is required to understand the stability and interaction between ligand and protein. This simulation is carried out by mimicking the property of the blood environment to get representative data. Molecular dynamic research on albumin had been carried out by Sudhamalla et al. [15] on $\beta$-sitosterol and albumin, and research by Artali et al. [16] concerning molecular dynamic simulation of binding sites in HSA. Research on docking interaction between metformin in HSA and glycated HAS (gHSA) was done by Rahnama et al. [17]. However, research on molecular dynamics simulation of HSA and gHSA with T2DM therapy drugs (metformin) and ASX has never been done. This study aims to compare the stability of metformin and ASX in gHSA and see its impact on albumin conformation.

\section{METHODS}

This research does not have any IRB/IACUC approval because there was no human, animal, or cell involvement. This research was purely in silico studies.

\section{Docking}

Data about the structure of the protein albumin (HSA) obtained from PDB site (http://www.rcsb.org/pdb) with PDB ID are 4K2C and then saved in a format (. pdb). Data about the ligand docking material compounds such as glucose, ASX, and metformin derived from PubChem database (https://pubchem.ncbi.nlm.nih.gov) with ID 79025, 5281224, and 4091, respectively. Water molecule and ligands were removed from HSA (4K2C) before docking with application Discovery Studio 2016 Client, and then, the result is stored as a file in the format (. pdb). Docking process using PyRx by paying attention to the binding affinity and searching box. All of the docking process by AutoDock Vina that built in PyRx using the same vina search box. Center of box is X: 11.5350, Y: -23.336, and Z: 5.6878 and the dimensions $(\AA)$ : X: 90.1439, Y: 109.1736, and Z: 79.3440. Searching box in AutoDock Vina was used to find the best location of ligand molecules binds into protein structure [18]. Discovery Studio is utilized analysis of receptor-ligand interactions.

\section{Molecular dynamics simulation}

YASARA is used for molecular dynamics simulation [19] with running time of $15.000 \mathrm{ps}$, and default physiological $\mathrm{pH}$ at 7.4 also the ion concentration as a mass fraction, here we use $0.9 \% \mathrm{NaCl}$. Temperature in the simulation was $310 \mathrm{~K}$, and because we used water for solvents (residues HOH) so the density is $0.997 \mathrm{~g} / \mathrm{l}$. The root mean square deviation (RMSD) and root mean square fluctuation (RMSF) value are determined from molecular dynamics simulation. Analysis of the protein backbone RMSD and the potential energy is obtained by running macro MD_Analyze, whereas the analysis of binding energy used the macro $m d_{\text {- }}$ analyzebindenergy, as for playing back the results of molecular dynamics simulation used macro $m d$ _play and recorded with application icecream Screen Recorder, output result was a file with extension (. wmv).

\section{RESULTS}

Pharmaceutical has known that the distribution as well as the efficacy and metabolism of drug greatly influenced by the affinity of drugs against the protein, especially HSA. Binding of HSA may change depending on condition and type of binding that is occurring [20]. Glycation is done on HSA using docking method by adding glucose on the protein (Fig. 1a). Binding affinity showed that astaxanthin to bond more easily with the gHSA and HSA than metformin. The binding affinity from astaxanthin to gHSA was $-8.9 \mathrm{kcal} / \mathrm{mol}$ while metformin to gHSA was $-5.0 \mathrm{kcal} / \mathrm{mol}$. Similar results also indicated by docking of ASX to HSA compared with metformin (Table 1).

The docking results of glucose to HSA show that there was unfavorable donor-donor bond located on GLN(B):459 and hydrogen bonds at SER(B):193, ARG(B):145, and ASP(B):108 (Fig. 1b). This unfavorable donor-donor bond at glutamine (B) shows that the interaction can cause protein damage which is shown in their stabilization when molecular dynamic was performed. Docking result from ASX-gHSA (Fig. 1c) and Fig. 1d) showed hydrogen bond at LYS(B): 212, and alkyl bonds at VAL(B): 325, PHE(B): 228, VAL(B): 482, LYS(B): 351, ARG(B): 348, LEU(B): 380, ALA(B): 350, LEU(B): 327, ALA(B): 213, VAL(B): 216, LEU(B): 331, and ARG(B): 209. The hydrogen bonds implied that these compound and protein were strongly bond. Free energies transfer of macromolecular complexes and biological macromolecules is one of hydrogen bonds function [21].

Docking result of ASX-HSA (Fig. 1e and f) showed hydrogen bonds at GLU(A): 492, VAL(A): 493 and alkyl bonds at ALA(A): 552, ALA(A): 406, ARG(A): 410, LYS(A): 413, LYS(A): 402, LYS(A): 545, MET(A): 548, and TYR(A): 401. The binding site of ASX in HSA is on A-side chain, while in gHSA is on B-side chain. The specific binding site of ASX to HSA can increase scavenging activity, while the specific binding site of ASX to gHSA is needed to prevent gHSA from further destabilizing.

Docking result of metformin-HSA (Fig. 2a and b) showed attractive charge bonds at ASP(A): 108 and GLU(A): 465, and hydrogen bonds at TYR(A): 148, LEU(A): 103, GLN(A): 104, and LYS(A): 106. The binding site of metformin in HSA is on A-side chain, while in gHSA is on B-side chain. Docking result of metformin-gHSA (Fig. 3a and b) showed attractive charge bond at SER(B): 579 and hydrogen bonds at ALA(B): 582, ALA(B): 539, and LEU(B): 532. There are unfavorable positive-positive bonds at LYS(B)536 and carbon-hydrogen bond at GLN(B): 580. Molecular dynamics simulations can provide additional knowledge about the condition of the dynamics that occur on a timescale that is different from the protein and ligand complexes in aqueous including $\mathrm{pH}$, temperature as well as the pressure of the solution used in the simulation.

The results of potential energy analysis by molecular dynamics simulation toward four simulations, which are albumin (HSA), gHSA, ASX-gHSA, and ASX-HSA showed stability at average 500 ps after simulation was started. Initiation state of simulation showed that HSA, gHSA, ASX-gHSA, ASX-HSA, metformin-HSA, and metformin-gHSA are $-2276091.991 \mathrm{~kJ} / \mathrm{mol},-2273555.427 \mathrm{~kJ} / \mathrm{mol}$, $-2262791.785 \mathrm{~kJ} / \mathrm{mol},-2270285.252 \mathrm{~kJ} / \mathrm{mol},-2276120.467 \mathrm{~kJ} / \mathrm{mol}$, and $-2278476.02 \mathrm{~kJ} / \mathrm{mol}$, respectively. Binding energy also showed the

Table 1: Binding affinity from the docking method

\begin{tabular}{llll}
\hline Protein & Ligand & Complex & $\begin{array}{l}\text { Binding affinity } \\
\text { (kcal/mol) }\end{array}$ \\
\hline HSA & Glucose & gHSA & -5.8 \\
gHSA & Astaxanthin & Astaxanthin-gHSA & -8.9 \\
HSA & Astaxanthin & Astaxanthin-HSA & -8.6 \\
gHSA & Metformin & Metformin- gHSA & -5.0 \\
HSA & Metformin & Metformin-HSA & -4.9 \\
\hline
\end{tabular}

HSA: Human serum albumin, gHSA: Glycated human serum albumin 


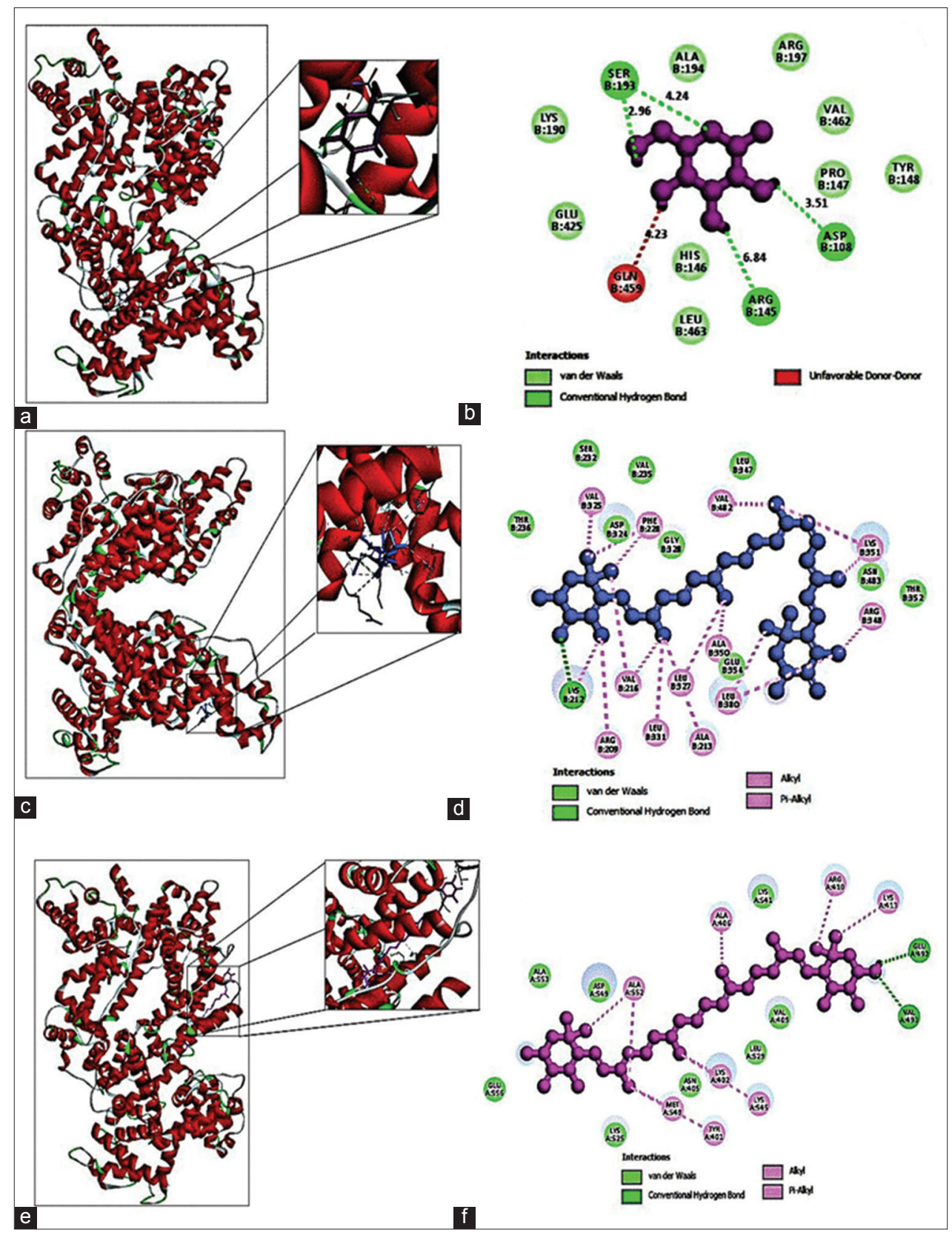

Fig. 1: Docking result (a) glycated human serum albumin (gHSA), (b) two-dimensional (2D) docking interaction of glucose-HSA, (c) astaxanthin (ASX)-gHSA, (d) 2D docking interaction of ASX-gHSA, (e) ASX-HSA, (f) 2D docking interaction of ASX-HSA

same pattern like potential energy but reach stabilization at 650 ps. Initiation state of simulation ( 0 ps) showed that HSA, gHSA, ASX-gHSA, ASX-HSA, metformin-HSA, and metformin-gHSA are $97982.872 \mathrm{~kJ} / \mathrm{mol}$, $-98017.753 \mathrm{~kJ} / \mathrm{mol}, 97102.554 \mathrm{~kJ} / \mathrm{mol}, 96684.082 \mathrm{~kJ} / \mathrm{mol},-97838.18 \mathrm{~kJ} / \mathrm{mol}$, and $98064.579 \mathrm{~kJ} / \mathrm{mol}$, respectively. When binding and potential energy of a simulation reach stability, it means that the molecular dynamics simulation data are valid.

On the simulation with time period at $15.000 \mathrm{ps}$, data showed that RMSD backbone RMSD (Fig. 4) of gHSA is unstable because at 2500 ps graphic has shown peak above $4 \mathrm{~nm}$, while the HSA normal shows instability on 5375 ps. Meanwhile, ASX-HSA shows the stability of the early stages of initiation until the end of the molecular dynamics simulation. ASXgHSA shows improvement of stability so much different compared to gHSA condition. These results indicate the occurrence of protein structure improvement from gHSA toward normal HSA condition. These improvements include the stability of binding interaction and less unfolding moment when the simulation was run.
Compared to metformin-gHSA shows instability at 4875 ps and continues to rise RMSD until the end of the simulation. It also shows that the unfolding process in albumin protein is replicated so that the bond between sides A and B becomes detached. RMSD result showed that metformin-HSA reached stability, but movie visualization result showed the release of metformin bonds on albumin while albumin did not experience unfolding event. So the RMSD result showed stability of albumin it self without metformin because it was released before molecular dynamic simulation end.

Analysis of RMSD was performed not only at protein backbone but also on residues. Fig. 5a-e showed a comparison between docking residues of HSA, gHSA, ASX-HSA, ASX-gHSA, metformin-HSA, and metformingHSA. Fig. 5a showed that residues of SER(B): 193, ASP(B): 108, ARG(B): 145, and GLN(B): 459 in HSA still under $4 \mathrm{~nm}$. Meanwhile, in gHSA, SER(B): 193 is $4.32 \mathrm{~nm}$ and GLN(B): 459 at $4.75 \mathrm{~nm}$. Fig. $5 \mathrm{~b}$ showed a comparison of docking residues between gHSA and ASX-gHSA. There 


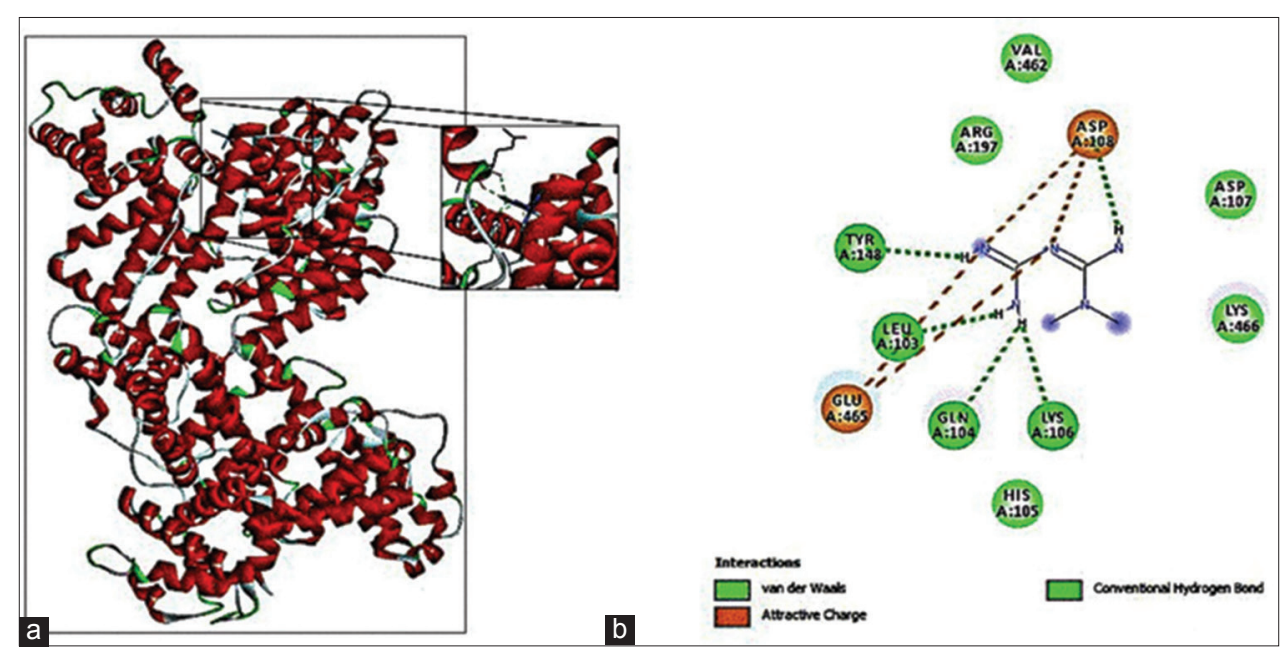

Fig. 2. Docking result (a) metformin-human serum albumin (has) (b) two-dimensional docking interaction of metformin-HAS

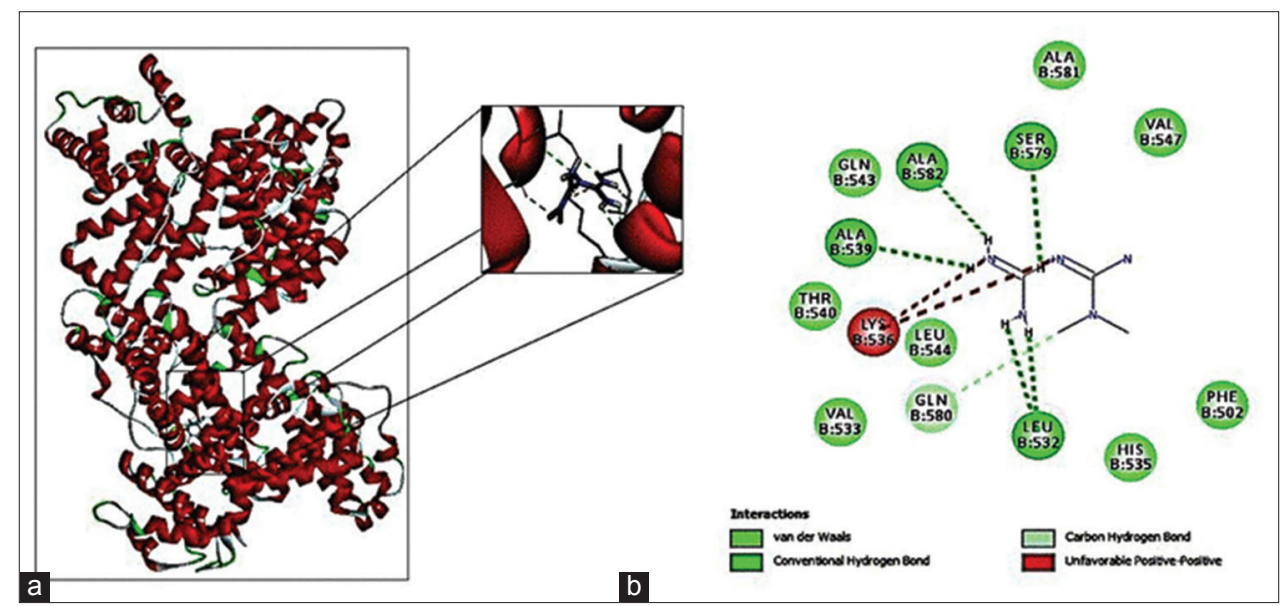

Fig. 3: Docking result (a) metformin-glycated human serum albumin (gHSA) (b) two-dimensional docking interaction of metformin-gHSA

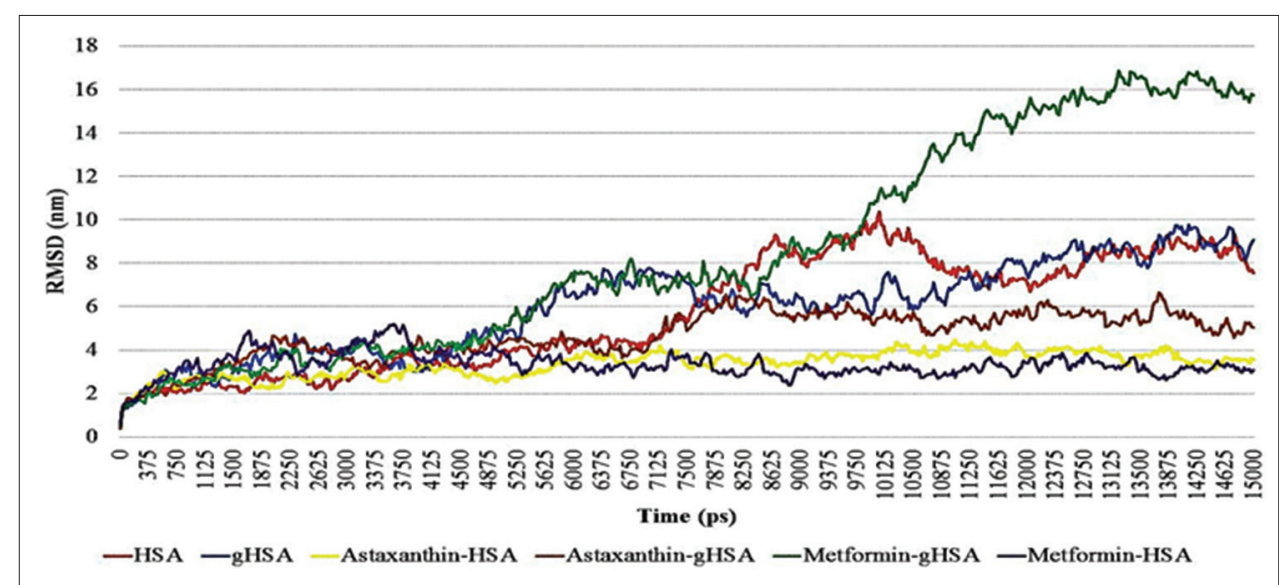

Fig. 4: Root mean square deviation backbone of human serum albumin (HAS) (red line), glycated HAS (gHSA) (blue line), astaxanthin (ASX)-HSA (yellow line), ASX-gHSA (brown line) also metformin-HSA (purple line), and metformin-gHSA (green line). Simulation of molecular dynamic was run in $\mathbf{1 5 . 0 0 0} \mathrm{ps}$

are 19 residues consisting of four residues from docking of gHSA and 12 residues from ASX binding to gHSA.

The data showed stability from ASX complex with gHSA like in residues VAL(B): 325, gHSA at $6.68 \mathrm{~nm}$, but when ASX was bound to gHSA, it became $3.74 \mathrm{~nm}$. Residues PHE(B): 228 also showed the change of RMSD from $8.45 \mathrm{~nm}$ in GHSA to $4.45 \mathrm{~nm}$ in ASX-gHSA. ASX also showed to be a stabilizer of energy in albumin normal (Fig. 5c). Stability also showed at metformin-HSA but, in this case, the bond between metformin and albumin had loose at 6875 ps so the RMSD of Fig. 5(d) did not represent RMSD of ligand and protein. In Fig. 5e showed that instability occurred in metformin-gHSA complex. RMSD showed above $20 \mathrm{~nm}$ which means the unfolding of gHSA happened as we can see in movie visualization result. RMSF residues showed the same pattern as 


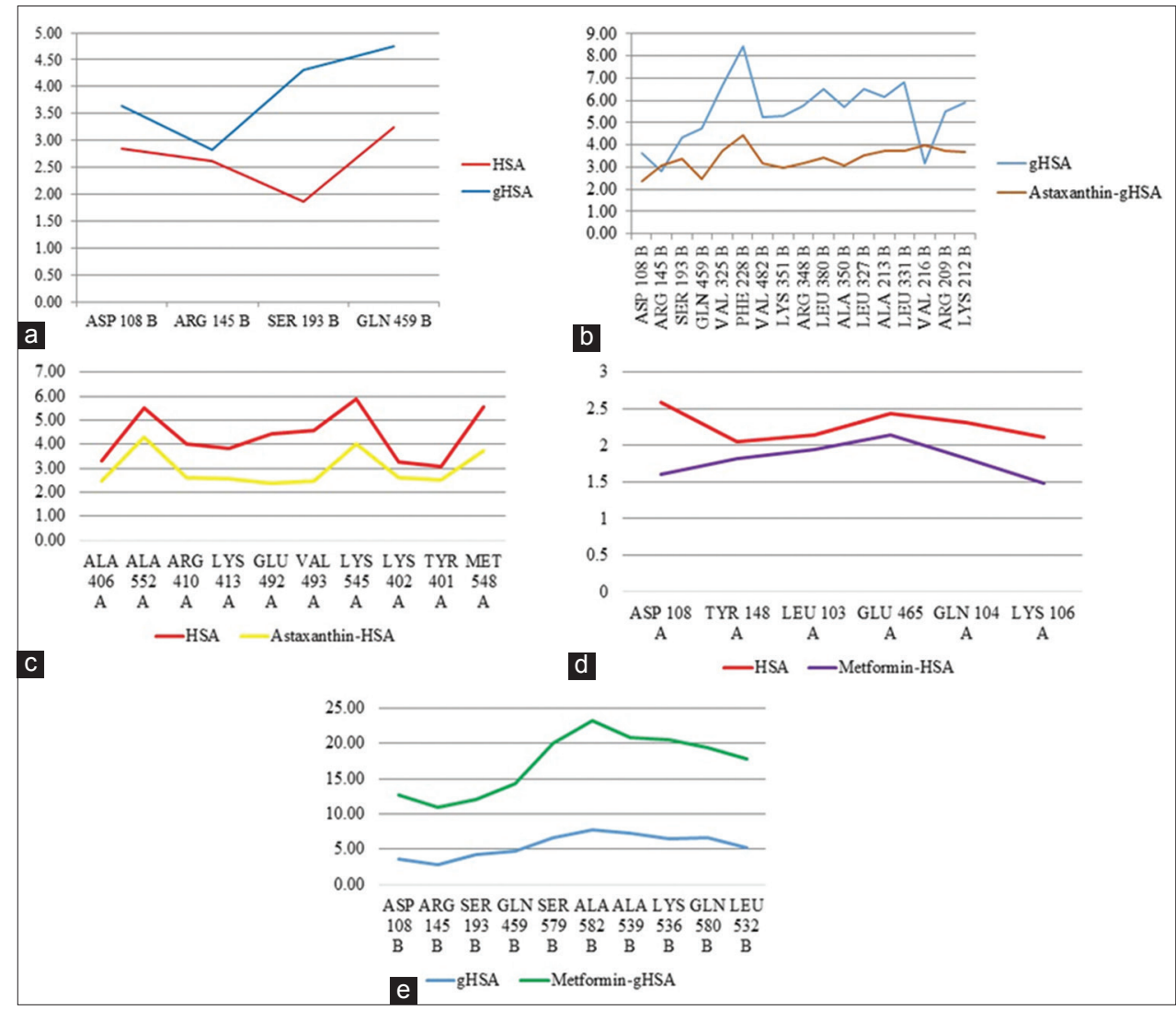

Fig. 5: (a-e) Root mean square deviation residues of human serum albumin (HSA) (red line), glycated HAS (gHSA) (blue line), astaxanthin (ASX)-HSA (yellow line), ASX-gHSA (brown line) also metformin-HSA (purple line), and metformin-gHSA (green line)
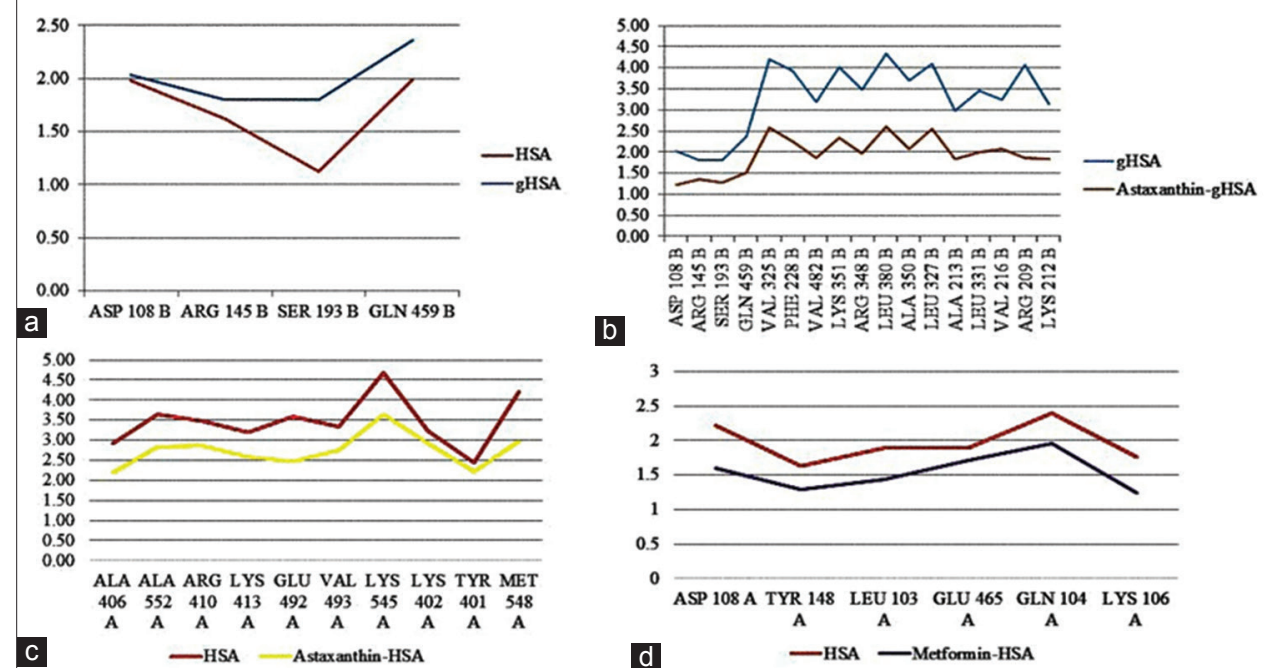

b
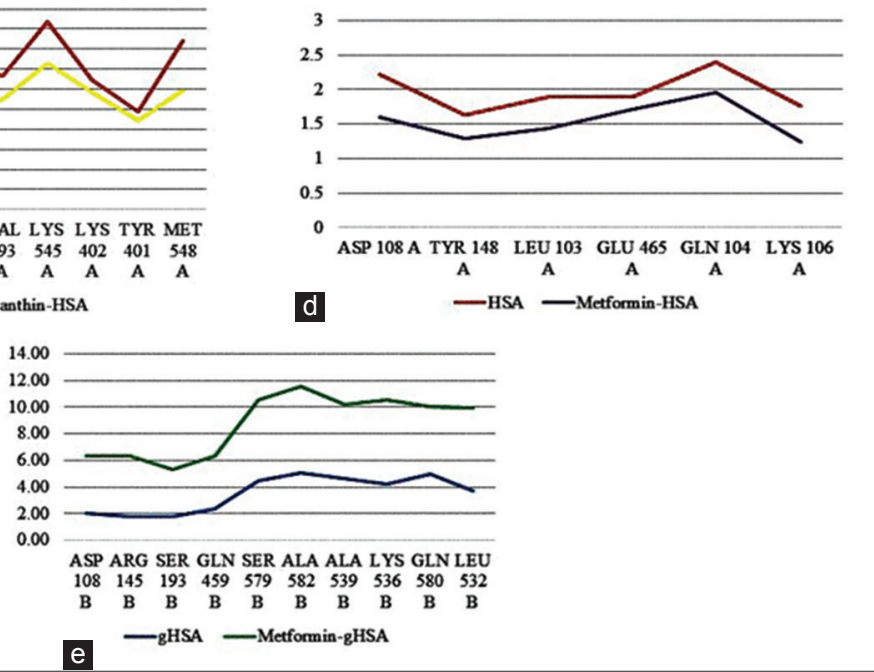

Fig. 6: (a-e) Root mean square fluctuation residues of human serum albumin (HSA) (red line), glycated HSA (gHSA) (blue line), astaxanthin (ASX)-HSA (yellow line), ASX-gHSA (brown line) also metformin-HSA (purple line), and metformin-gHSA (green line) 
RMSD residues (Fig. 6). The stability between glycated albumin with and without astaxanthin is shown in Fig. $6 \mathrm{~b}$ and c.

Visualization of molecular dynamic simulation (Fig. 7) showed the last state in 15000 ps. Sidechain A of HSA was colored in blue and green and is sidechain B. ASX can bound into sidechain B where glycation was happened. There was some noticeable unfolding happened in Fig. $7 \mathrm{~b}$ which is gHSA.

However, the other three conditions (HSA, ASX-HSA, and ASX-gHSA) on the bond between the two side chains still strong until the end of the simulation. This indicates that ASX can help the stability of the glycated albumin complex molecules or normal compared to metformin. While on metformin-gHSA, the bond between the A and B side of protein at 6750 ps be the initial time of bound released. This happens until the end of molecular dynamics simulation and then on metformin-HSA showed the release of bonds between metformin with HSA protein in $6800 \mathrm{ps}$

\section{DISCUSSION}

Several molecular dynamics simulations have been performed to study human blood albumin. Fujiwara and Amisaki [22] analyzed the molecular dynamics simulation between HSA and fatty acids, which high and low binding affinity and fatty acids on the albumin in the blood that has a positive correlation with the laboratory experiments. This means molecular dynamics simulation can be used to actual state description in the human body.

Based on these results, it showed that ASX can be a stabilizer of gHSA and HSA from the initial time to the end of molecular dynamics simulation. Nishigaki et al. [14] stated that ASX can reduce the cytotoxicity caused by glycated protein, especially by reducing lipid
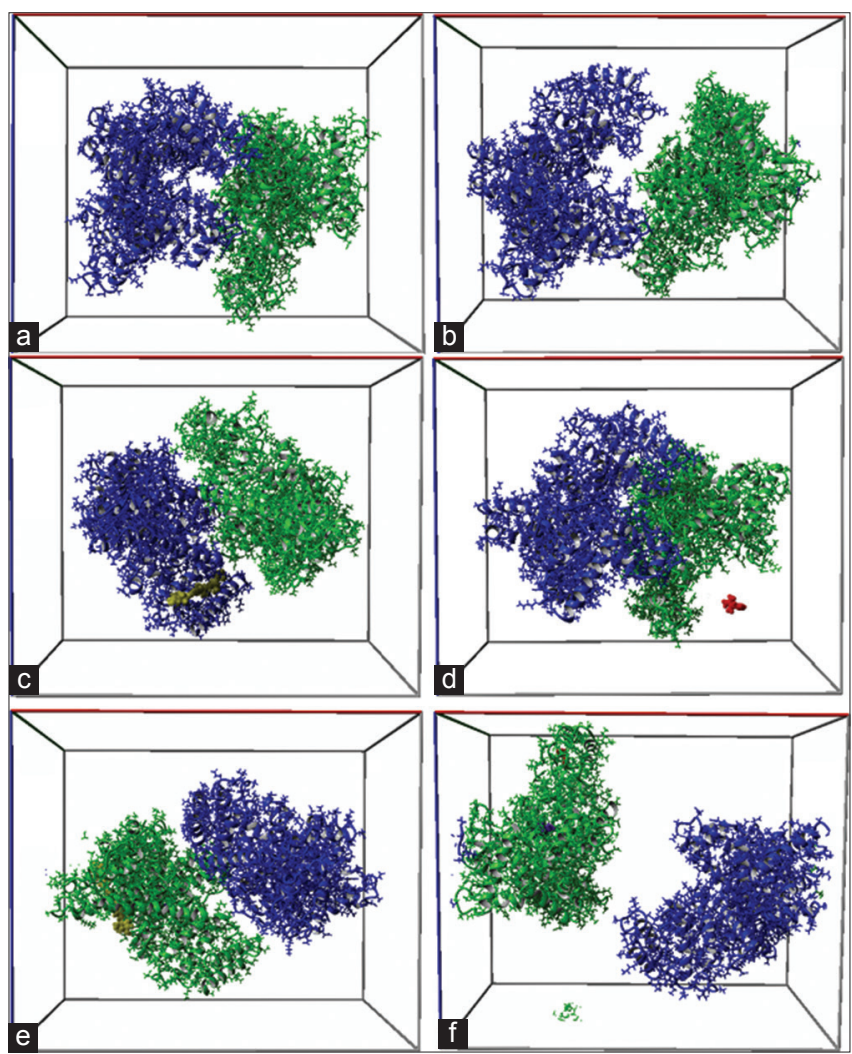

Fig. 7: (a-f) Molecular dynamic simulation at 15000 ps (a) human serum albumin (HSA), (b) glycated HSA (gHSA), (c) astaxanthin (ASX)-HSA, (d) metformin-HSA, (E) ASX-gHSA, and (F) metformingHSA. There are two sidechains of human albumin, sidechain A (blue) and sidechain B (green) identified by YASARA peroxidation. During the cooling of the $\mathrm{O}_{2}$, the energy was moved from $\mathrm{O}_{2}$ to the ASX molecule, turning it into rich energy of triplet state. Triplet state in ASX can return to the ground state by eliminating energy as heat or with physical cooling, leave the molecule ASX intact and ready for the next cooling events. This indicates that ASX is a stable compound that after performed scavenging activity, it is not being a free radical.

The instability of metformin in binding with glycated albumin and normal albumin can be due to the time spent in this study reaching 15000 ps. The aim of the researchers was to see stability in the long run, whereas in the research conducted by Artali et al. [16], it was shown that albumin was stable up to 2000 ps. The results showed that ASX was safe in long-term use because it remained attached to albumin both glycated and uncovered and maintained the protein conditions of both RMSD and RMSF. While the administration of metformin can cause unfolding events when docking with glycated albumin for the long term and also the release of docking bonds complex between metformin it self on metformin-HSA complex.

Docking between metformin and albumin has also been done by Rahnama et al. [17], the results of this study show that the binding between metformin and HSA has a stronger financial strength compared to gHSA. These results were obtained from analyzes using binding sites analysis with fluorescence and data from anisotropy spectroscopy. These results support this study that showed initial stability of metformin given to normal albumin more stable when compared to gHSA. The study also shows structural changes in HSA when it is docked with metformin, which is caused reduced of resistance against drug aggregation. The use of metformin itself has several side effects such as vomiting and nausea; these cases reach $20-30 \%$. The next side effect is lactic acidosis, where the incident average is $1 / 30,000$ cases. Most occur in T2DM patients with kidney and liver dysfunction [23]. Cases of death due to lactic acidosis conditions associated with the use of metformin occur quite a lot, in 2007-2008, there were 25 cases of severe lactic acidosis in the Swedish region, whereas in 2008 in America there were 21 fatalities related to the use of metformin. Furthermore, it happened in Italy from 2005 to 2010, there were 49 cases of lactic acidosis which were also related to the use of metformin with a total of 11 deaths [24]. Although it has side effects like the one above, metformin is also proven to help control blood sugar content in T2DM patients. The use of metformin wisely is the key to preventing these side effects.

\section{CONCLUSION}

ASX can be a novel therapeutic agent for T2DM in accordance to prevent gHSA from unfolding and maintain stability which showed by molecular dynamics simulation data. HSA and gHSA binding to ASX shows stability from the beginning to the end of the molecular dynamics simulation indicated by the values of RMSD backbone, residual RMSD (RMSDr), and residual RMSF (RMSFr), whereas the gHSA bound to metformin experiences unfolding event and loose bond between sites A and B at gHSA. The release of metformin with normal HSA bonds occurs at 6800 ps until the end of the simulation. Further laboratory experiments are now undergoing in our laboratory.

\section{ACKNOWLEDGMENTS}

Gratitude is also sent to Prof. Widodo, S.Si., M.Si., Ph.D.Med.Sc (widodo@ ub.ac.id) from the Biology Department, Brawijaya University, for allowed the researcher to used YASARA application. This research was supported by PMDSU and Ristek Dikti (PDUPT).

\section{AUTHORS' CONTRIBUTION}

All authors have their respective contributions to this journal, namely, Syahputra Wibowo carried out the experiment and wrote the manuscript, Sutiman Bambang Sumitro conceived the original idea and supervised the project, Sri Widyarti supervised the project, Akhmad Sabarudin supervised the project, and Djoko Wahono Soeatmadji supervised the project. 


\section{CONFLICTS OF INTEREST}

No potential conflicts of interest relevant to this article were reported.

\section{REFERENCES}

1. Bullon P, Newman HN, Battino M. Obesity, diabetes mellitus, atherosclerosis and chronic periodontitis: A shared pathology via oxidative stress and mitochondrial dysfunction? Periodontol 2000 2014;64:139-53.

2. Murray RK, Granner DK, Mayes PA, Rodwell VW. Harper's Illustrated Biochemistry. 26 $6^{\text {th }}$ ed. Boston: McGraw Hills Companies; 2003. p. 609-12.

3. Roche M, Rondeau P, Singh NR, Tarnus E, Bourdon E. The antioxidant properties of serum albumin. FEBS Lett 2008;582:1783-7.

4. Dobson CM. The structural basis of protein folding and its links with human disease. Philos Trans R Soc Lond B Biol Sci 2001;356:133-45.

5. Otagiri M, Chuang VT. Albumin in Medicine: Pathological and Clinical Applications. Singapore: Springer Nature; 2016.

6. Merlot AM, Kalinowski DS, Richardson DR. Unraveling the mysteries of serum albumin-more than just a serum protein. Front Physiol 2014;5:299

7. Barnaby OS, Cerny RL, Clarke W, Hage DS. Comparison of modification sites formed on human serum albumin at various stages of glycation. Clin Chim Acta 2011;412:277-85.

8. Arasteh A, Farahi S, Habibi-Rezaei M, Moosavi-Movahedi AA. Glycated albumin: An overview of the in vitro models of an in vivo potential disease marker. J Diabetes Metab Disord 2014;13:49.

9. Hirst JA, Farmer AJ, Ali R, Roberts NW, Stevens RJ. Quantifying the effect of metformin treatment and dose on glycemic control. Diabetes Care 2012;35:446-54.

10. Holman R. Metformin as first choice in oral diabetes treatment: The UKPDS experience. Journ Annu Diabetol Hotel Dieu 2007;13:13-20.

11. Grisouard J, TimperK, Radimerski TM, Frey DM, Peterli R, Kola B, et al. Mechanisms of metformin action on glucose transport and metabolism in human adipocytes. Biochem Pharmacol 2010;80:1736-45.

12. Woo SL, Xu H, Li H, Zhao Y, Hu X, Zhao J, et al. Metformin ameliorates hepatic steatosis and inflammation without altering adipose phenotype in diet-induced obesity. PLoS One 2014;9:e91111.
13. Wang YW, He SJ, Feng X, Cheng J, Luo YT, Tian L, et al. Metformin: A review of its potential indications. Drug Des Devel Ther 2017;11:2421-9.

14. Nishigaki I, Rajendran P, Venugopal R, Ekambaram G, Sakthisekaran D, Nishigaki Y, et al. Cytoprotective role of astaxanthin against glycated protein/iron chelate-induced toxicity in human umbilical vein endothelial cells. Phytother Res 2010;24:54-9.

15. Sudhamalla B, Gokara M, Ahalawat N, Amooru DG, Subramanyam R. Molecular dynamics simulation and binding studies of beta-sitosterol with human serum albumin and its biological relevance. J Phys Chem B 2010;114:9054-62.

16. Artali R, Bombieri G, Calabi L, Del Pra A. A molecular dynamics study of human serum albumin binding sites. Farmaco 2005;60:485-95.

17. Rahnama E, Mahmoodian-Moghaddam M, Khorsand-Ahmadi S, Saberi MR, Chamani J. Binding site identification of metformin to human serum albumin and glycated human serum albumin by spectroscopic and molecular modeling techniques: A comparison study. J Biomol Struct Dyn 2015;33:513-33

18. Jayanti GE, Widyarti S, Sabarudin A, Sumitro SB. Egg white albumin form complex with aspirin and caffeine and its role as free radical scavenger. Asian J Pharm Clin Res 2018;11:340-4.

19. Krieger E, Vriend G. YASARA view - molecular graphics for all devices - from smartphones to workstations. Bioinformatics 2014;30:2981-2.

20. Yue Y, Chen X, Qin J, Yao X. Characterization of the mangiferin-human serum albumin complex by spectroscopic and molecular modeling approaches. J Pharm Biomed Anal 2009;49:753-9.

21. Verma R, Jatav VK, Sharma S. Identification of inhibitors of dengue virus (DENV1, DENV2 and DENV3) NS2B/NS3 serine protease: A molecular docking and simulation approach. Asian J Pharm Clin Res 2015;8:287-92.

22. Fujiwara S, Amisaki T. Identification of high affinity fatty acid binding sites on human serum albumin by MM-PBSA method. Biophys $\mathrm{J}$ 2008;94:95-103.

23. Salpeter SR, Greyber E, Pasternak GA, Salpeter EE. Risk of fatal and nonfatal lactic acidosis with metformin use in Type 2 diabetes mellitus. Cochrane Database Syst Rev 2010;4:CD002967.

24. Vecchio S, Protti A. Metformin-induced lactic acidosis: No one left behind. Crit Care 2011;15:107. 